\title{
Distribution and pressure of active Lévy swimmers under confinement
}

\author{
Tingtao Zhou ${ }^{1}$, Zhiwei Peng' ${ }^{2}$, Mamikon Gulian ${ }^{3}$, and John F. \\ $\operatorname{Brady}^{1,2}$ \\ ${ }^{1}$ Division of Engineering and Applied Science, California Institute of Technology, \\ Pasadena, California 91125, USA \\ 2 Division of Chemistry and Chemical Engineering, California Institute of \\ Technology, Pasadena, California 91125, USA \\ ${ }^{3}$ Center for Computing Research, Sandia National Laboratories, Albuquerque, New \\ Mexico 87123, USA
}

Mar 2021

\begin{abstract}
Many active matter systems are known to perform Lévy walks during migration or foraging. Such superdiffusive transport indicates long-range correlated dynamics. These behavior patterns have been observed for microswimmers such as bacteria in microfluidic experiments, where Gaussian noise assumptions are insufficient to explain the data. We introduce active Lévy swimmers to model such behavior. The focus is on ideal swimmers that only interact with the walls but not with each other, which reduces to the classical Lévy walk model but now under confinement. We study the density distribution in the channel and force exerted on the walls by the Lévy swimmers, where the boundaries require proper explicit treatment. We analyze stronger confinement via a set of coupled kinetics equations and the swimmers' stochastic trajectories. Previous literature demonstrated that power-law scaling in a multiscale analysis in free space results in a fractional diffusion equation. We show that in a channel, in the weak confinement limit active Lévy swimmers are governed by a modified Riesz fractional derivative. Leveraging recent results on fractional fluxes, we derive steady state solutions for the bulk density distribution of active Lévy swimmers in a channel, and demonstrate that these solutions agree well with particle simulations. The profiles are non-uniform over the entire domain, in contrast to constant-in-thebulk profiles of active Brownian and run-and-tumble particles. Our theory provides a mathematical framework for Lévy walks under confinement with sliding no-flux boundary conditions and provides a foundation for studies of interacting active Lévy swimmers.
\end{abstract}

Keywords: active matter, Lévy walk, confinement, fractional PDE

Submitted to: J. Phys. A: Math. Theor. 


\section{Introduction}

Active matter refers to systems of self-propelled particles or swimmers, such as motile cells or synthetic Janus particles. In contrast to passive Brownian systems, active particles exhibit intriguing behaviors including accumulation at confining boundaries, upstream swimming in Poiseuille flow, and motility-induced phase separation [1 3]. The non-equilibrium nature of active matter poses a number of theoretical challenges and has attracted considerable interest in the past few decades. To aid in the understanding and prediction of the dynamics of active matter, various theoretical models have been proposed, either at the particle scale [2] or the macroscopic scale [4, 5]. Among them, stochastic dynamical models such as active Brownian particles (ABP) and run-andtumble particles (RTP) are widely used [1, 2, 6]. In these stochastic models, an active particle self-propels with an intrinsic speed $U_{0}$ and reorients on a timescale $\tau$ due to either continuous rotary diffusion (ABP) or discrete tumbling events (RTP). For ABPs and RTPs, the underlying reorientation is a Gaussian or Markovian process, respectively. Other variants in this family such as active Ornstein-Uhlenbeck particles (AOUP) [7, 8] have been proposed as well. The directed swimming motion persists at short times $(t \ll \tau)$ during which one observes ballistic motion of an individual particle. At times much larger than $\tau$, the swimming motion becomes a random walk characterized by the so-called swim diffusivity, $D^{\text {swim }} \sim U_{0}^{2} / \tau$.

However, many active biological systems such as albatrosses [9], bumblebees and deer [10], bacteria [11 14], soil ameoba [15], fungi [16], T cells [17], as well as humans [1820], are known to exhibit super-diffusive behavior at long times described by Lévy processes [21 23]. Hydrodynamics of active suspensions can also induce Lévy motion of passive tracers [24]. In particular, recent experimental works have shown that, under certain conditions, Escherichia coli (E. coli) can travel comparatively large distances that exceed predictions from the RTP model with exponentially-distributed run-times [11, 13]. Instead, a power-law distribution of run-times that leads to Lévy motion has been shown to explain the observed persistent motion [13]. Lévy motions are understood as a more advantageous foraging or search strategy than Brownian motion in many settings [25 30], leading to applications in robotics and artificial intelligence for better search pattern design [31 34$]$.

As pointed out by Zaburdaev et al. [23], when discussing Lévy motion, it is important to distinguish Lévy walks from the earlier mathematical model of Lévy flights. The latter, by definition, are jump processes with unbounded velocity magnitudes. At each time interval, a spatial jump is drawn from an appropriate heavy-tailed distribution, leading to discontinuous-in-time paths in the continuum limit. Such models violate Einstein's locality principle in physics, and the nature of instantaneous jumps make it impractical, if not impossible, to deal with in interacting systems. The resolution of instantaneous jumps in overdamped Langevin dynamics (of ABP, RTP, AOUP) was discussed by Fodor et al. [35], while active matter models based on Lévy flights were proposed in Cairoli and Lee [36]. On the other hand, Lévy walks involve particles 
that change direction at random times, but travel in each direction with bounded (e.g., constant) velocity for a persistent time sampled from a heavy-tailed distribution. Such motion can be thought of as interpolation between ballistic motion and Brownian motion. Fractional Brownian motion (FBM) has also been studied [37 40] in parallel to the Lévy walk model. FBM shares many similar behaviors with Lévy walks, but it is constructed from correlated noise and falls within the framework of Gaussian processes.

In general, the continuum limit of Lévy processes is described by fractionalorder calculus. Since the first attempt on fractional calculus by Leibniz in 1695, mathematicians have developed this idea into a rich field where definitions for differentiation and integration abound [41, 42], generalizing the concept of integerorder calculus in different ways. A fundamental connection between fractional calculus and Lévy processes is suggested by the generalization of the central limit theorem (CLT) [43 46], in which the average of independent, identically distributed (i.i.d.) random variables with possibly infinite variance but power-law (Paretian) tails converge to $\alpha$-stable distributions [44, 47]. The Gaussian distribution is the special case of finite variance statistics, corresponding to the Wiener (Brownian) process. Comparing the characteristic function of an $\alpha$-stable distribution with a Gaussian one then hints at the more general formula of a fractional derivative in Fourier space, leading to the Riesz fractional derivative. The development of the continuous-time random walk (CTRW) framework [48] and later Lévy walks as a more physical alternative to Lévy flights [23] lead a wide variety of proposed fractional-order models, especially in bounded domains. However, in many studies, the form of fractional PDEs were hypothesized based on intuition or convenience rather than a rigorous derivation, and explicit treatment of boundaries are often left out.

In many biological and industrial processes, such as infection by motile bacteria or the formation of biofilms, active swimmers are confined by a boundary. An active particle with a constant swim speed that collides with a wall keeps pushing against the wall and sliding along it, and is able to swim away only after a reorientation event that turns it into the bulk. This steric interaction leads to a boundary accumulation layer of active particles at the wall, whereas passive Brownian particles exhibit a uniform density distribution in the entire domain. This behavior has been exploited to design several interesting mechanical systems including microscale gears powered by motile bacteria suspensions [49] and mechanical traps used to collect bacteria from the surrounding fluid [50 52]. In the presence of flow, such as in the human urinary tract and medical catheters, the vorticity of the flow orients the particles in the accumulation boundary layer to pointing upstream, and this upstream swimming of bacteria often leads to infection [53, 54]. Nano-robots [55] usually work in confined geometries, especially in porous networks such as human blood vessels. Better understanding of the density distribution in these bounded geometries can assist the design of devices and robots, further advancing environmental or medical engineering applications. Previous literature has considered confinement effects of ABPs [56] and RTPs [57, 58], where the non-uniform steady state density across a channel is due to boundary layer 
accumulations.

Motivated by the recent biological and microfluidic experiments [11 14], as well as progress in derivations of fractional PDE's from stochastic kinetics of Lévy walks in unbounded domains [59, 60], here we propose a model of micro-swimmers that perform Lévy walks in bounded domains, and refer to these swimmers as active Lévy swimmers. Whereas the Lévy walk model only concerns the trajectory of a single walker, our active Lévy swimmer model can incorporate physical interactions among the swimmers at finite packing density and with other objects as well as with the confining geometry. Moreover, while elastic reflecting boundary conditions have been considered for Lévy walks in 1D [61], leading to uniform bulk density distributions at steady-state, we impose hard sphere interactions that are widely used to model active swimmers, leading to sliding no-flux boundary conditions.

In this first work, we consider the ideal case where the swimmers do not interact with each other, but only with the walls. We derive a full set of transport equations for the probability density function of the Lévy swimmers, and analyze the limits of weak and strong confinement. In the weak confinement limit, we show that these equations reduce to a fractional-order diffusion equation with sliding no-flux boundary conditions for the number density. We report U-shaped bulk density distributions at steady state, in contrast to the constant-in-the-bulk profiles of ABPs and normal RTPs. Our theory provides a foundation for studies of interacting active Lévy swimmers.

\section{The model}

As shown in Figure 1, an active Lévy swimmer is a sphere that propels itself with a constant swim force. We neglect hydrodynamic interactions. In Stokes flows, inertia is negligible and this results in a constant swim speed $U_{0} \boldsymbol{q}$ when not interacting with other swimmers or with a wall, where $\boldsymbol{q}$ is the unit vector representing the orientation. The swimmer changes the orientation of the swim force - hence the velocity direction $\boldsymbol{q}$ - stochastically, a behavior referred to as tumbling. The run-time $\tau$ between two consecutive tumbles is sampled from a type-II Pareto distribution (also known as the Lomax distribution [62, 63] )

$$
\psi(\tau)=\frac{\alpha \tau_{0}^{\alpha}}{\left(\tau+\tau_{0}\right)^{\alpha+1}},
$$

where $\tau_{0}$ is a characteristic timescale. It is known that when $0<\alpha<1$ the mean runtime $\tau_{m}$ diverges and Lévy walks in this regime display weak ergodicity breaking [63], so that the time average differs from the ensemble average. We will restrict our model of Lévy swimmers to $1<\alpha<2$; in this case, the mean run-time $\tau_{m}$ is finite and related to the characteristic timescale via

$$
\tau_{m}=\frac{\tau_{0}}{\alpha-1}
$$

The $\alpha>2$ regime is expected to converge to the Brownian limit since the variance $\sigma^{2}$ is finite and is given by $\sigma^{2}=\tau_{m}^{2} \alpha /(\alpha-2)$. We compare the results from Lévy 


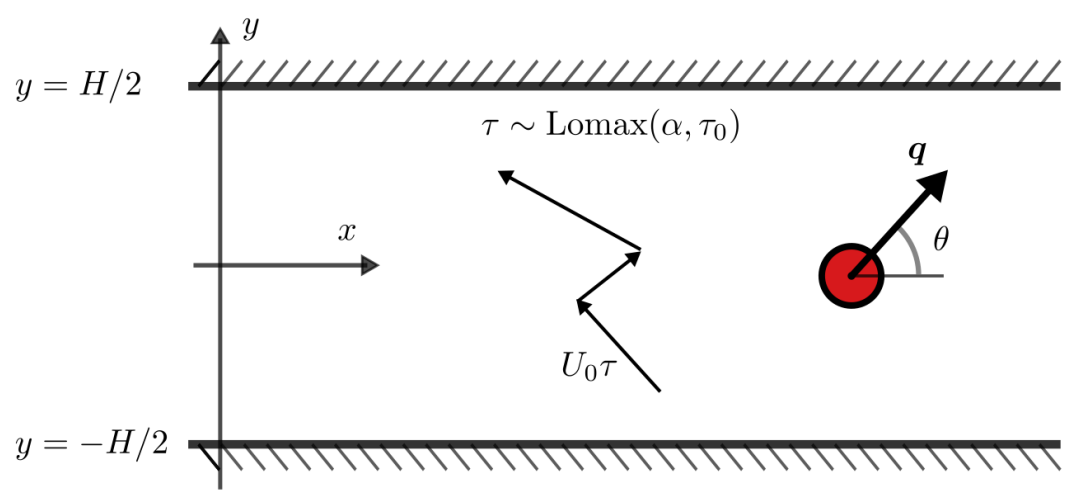

Figure 1. Schematic of a Lévy swimmer confined in a $2 \mathrm{D}$ channel. The run-time $\tau$ follows a Lomax distribution, featuring a power-law tail. When the swimmer encounters a wall, it will maintain its orientation and slide along the wall before tumbling.

swimmers with those of $\alpha=3>2$, using the later case to represent Gaussian models by appealing to the CLT. When a Lévy swimmer collides with another swimmer, it is constrained by hard sphere repulsions. When it collides with a wall, it is constrained by hard wall repulsion and remains free to slide along the wall, in a similar way to a Skorokhod boundary condition for Brownian motion [64, 65]. Specific details of the particle simulations used in this article are given in Appendix A

For simplicity, we perform analysis for non-interacting Lévy swimmers confined by walls of a channel. We analyze the density distribution and forces exerted on the walls through kinetic equations, and rigorously prove a fractional diffusion equation governing the steady state density in the weak confinement limit by extending the works of Refs. [59, 60, 66]. Our results are compared to particle simulations. In Section 5, we show that at steady state, Lévy swimmers maintain a U-shaped bulk distribution even at weak confinement, in contrast to the accumulation boundary layer effect of ABPs and RTPs, which scales with activity, manifesting the fundamental difference between Lévy processes (fractional-order) and Gaussian processes (integer-order).

\section{Transport equations for ideal Lévy swimmers in a channel}

The stochastic process of Lévy swimmers can be described by a set of transport equations for the particle probability distribution. There are two features that are noteworthy compared with the ABP/RTP model. First, there is no explicit translational diffusion; as a result, we expect singular accumulation of the particles on the boundaries, as would occur even for normal RTP with exponentially distributed run-times [58]. Second, both ABP and normal RTP are essentially Markovian and lose memory at the long-time limit. However, this Markovian property is lost for Lévy swimmers given the slow power-law decay of their run-time distribution. Hence, we have to explicitly keep track of the 
current run-time variable of the Lévy swimmer, denoted by $\tau[60,67]$. With these in mind, we can write down the kinetics in 2D. The distribution of particles inside the channel of width $H$ is governed by

$$
\left(\frac{\partial}{\partial \tau}+\frac{\partial}{\partial t}+U_{0} \boldsymbol{q} \cdot \nabla\right) P(x, y, t, \tau, \theta)=-\beta(\tau) P(x, y, t, \tau, \theta),
$$

where $\boldsymbol{q}=(\cos \theta, \sin \theta)$ is the unit vector for particle orientation, and $-H / 2<y<H / 2$. The term $\beta(\tau)$ is the tumbling rate function, which is generally a function of the current run-time $\tau$. For active Lévy swimmers it is given by

$$
\beta(\tau)=\frac{\alpha+1}{\tau+\tau_{0}}
$$

In the case of normal RTP, $\beta=1 / \tau_{m}$, a constant, and equation (3) reduces to the Smoluchowski equation (10), as shown below.

This equation can be understood by considering an infinitesimal time interval $d t$ : the probability density $P$ is shifted in space-time by the material derivative $\left(\partial / \partial t+U_{0} \boldsymbol{q} \cdot \nabla\right) P d t$. For all swimmers that did not tumble during $d t$, their current run-time increases by $d \tau=d t$, hence the term $\partial P / \partial \tau$ in equation (3). A fraction of the swimmers $\beta(\tau) P d t$ tumbled to other directions during $d t$, giving the sink term on the RHS of equation (3). All of these swimmers that just tumbled now have their current run-time renewed to be $\tau=0$, hence giving the initial condition for $\tau$,

$$
P(x, y, t, 0, \theta)=\frac{1}{2 \pi} \int_{0}^{t} \beta\left(\tau^{\prime}\right) d \tau^{\prime} \int_{0}^{2 \pi} P\left(x, y, t, \tau^{\prime}, \theta^{\prime}\right) d \theta^{\prime}
$$

Similarly, for the probability density on the upper wall, we have

$$
\frac{\partial \phi^{+}}{\partial \tau}+\frac{\partial \phi^{+}}{\partial t}+U_{0} \cos \theta^{+} \frac{\partial \phi^{+}}{\partial x}=-\beta(\tau) \phi^{+}+j_{b}^{+},
$$

where $\phi^{+}$is short for the number density $\phi$ on the wall at the upper wall $y=H / 2$,

$$
\phi^{+}=\phi(x, y=H / 2, t, \tau, \theta) .
$$

The additional source term $j_{b}^{+}$is the net flux of swimmers going from the bulk into the upper wall at $y=H / 2$

$$
j_{b}^{+}=U_{0} P\left(x, y=H / 2, t, \tau, \theta^{+}\right) \sin \left(\theta^{+}\right)
$$

The equation for the lower wall at $y=-H / 2$ is symmetric to that of the upper wall and is omitted here. The superscripts on $\theta^{ \pm}$is a reminder of $0<\theta^{+}<\pi$ and $-\pi<\theta^{-}<0$.

The bulk density flux close to the wall is coupled with the swimmer flux tumbling from the wall through

$$
\int_{0}^{t} \beta(\tau) d \tau \frac{1}{2 \pi} \int_{0}^{\pi} d \theta^{\prime} \phi^{+}=\phi^{+}\left(x, t, 0, \theta^{+}\right)=-P\left(x, y=H / 2, t, 0, \theta^{-}\right) U_{0} \sin \theta^{-} .
$$


In this setting, the only parameter for RTPs is the strength of confinement $\ell / H$, where $\ell=U_{0} \tau_{m}$ is the average run length. For Lévy swimmers there is another parameter, the power-law index $\alpha$ of run-time distributions, independent of the average run-time $\tau_{m}$. At steady state, equations (31)-(9) can be further simplified; see Appendix B.

We note that one can recover the equations for normal RTPs from the above general kinetic equations (3-6). In that case $\beta(\tau)=\beta=1 / \tau_{0}$ is a constant, hence integration over $\tau$ is trivial and gives

$$
\begin{aligned}
\left(\frac{\partial}{\partial t}+U_{0} \boldsymbol{q} \cdot \nabla\right) p(\boldsymbol{r}, t, \theta) & =\frac{1}{2 \pi} \int_{0}^{2 \pi} p\left(\boldsymbol{r}, t, \theta^{\prime}\right) d \theta^{\prime}-\beta p(\boldsymbol{r}, t, \theta)-P\left(\boldsymbol{r}-U_{0} t \boldsymbol{q}, 0,0, \theta\right) e^{-\beta t} \\
& =\mathcal{L} p-P\left(\boldsymbol{r}-U_{0} t \boldsymbol{q}, 0,0, \theta\right) e^{-\beta t}
\end{aligned}
$$

where

$$
p(\boldsymbol{r}, t, \theta)=\int_{0}^{t} P(\boldsymbol{r}, t, \theta, \tau) d \tau,
$$

is the reduced probability density, and the turning operator $\mathcal{L}$ is defined [68, 69] by

$$
\mathcal{L} p=\frac{1}{2 \pi} \int_{0}^{2 \pi} p\left(\boldsymbol{r}, t, \theta^{\prime}\right) d \theta^{\prime}-\beta p(\boldsymbol{r}, t, \theta) .
$$

In the long time limit, the last term of equation (10) represents the exponentially vanishing initial condition, and is usually omitted in multiscale analysis of RTPs.

\section{Limit of strong confinement: no time to tumble in the bulk}

When $\ell / H \gg 1$, the confinement is very strong, so the swimmers barely tumble in the bulk of the channel. Therefore, one expects the swimmers to contribute equally to the bulk density profile at each point in space since they merely cross the channel width with a constant speed.

In $1 \mathrm{D}$ this is asymptotically true for both Lévy and normal swimmers when their average run-time $\tau_{m}$ is longer than the channel crossing time $t_{c}=H / U_{0}$. As shown in Figure 2, the simulated bulk density becomes almost flat in the strong confinement limit for which $\ell / H>1$. In Figure 2 (as in Figure 3), each simulation curve integrated over the interior of the channel gives the corresponding bulk fraction of swimmers, not including the singular accumulation on the walls. In the strong confinement limit the fraction of swimmers accumulated exactly on the wall can be estimated by time average of the individual trajectories, noting that we restrict the power-law index $\alpha>1$ so that time average equals the ensemble average [63]. In $1 \mathrm{D}$, the time spent inside the bulk is a constant $t_{c}$. The fraction of swimmers in the bulk is then $f_{\text {bulk }}=H / \ell$. Since in 1D the swimmer only takes orientations of either to the left or to the right, this partitioning between bulk and boundary immediately translates into the force on the wall, as in equation (13). As the confinement strength $\ell / H$ increases, larger fractions 

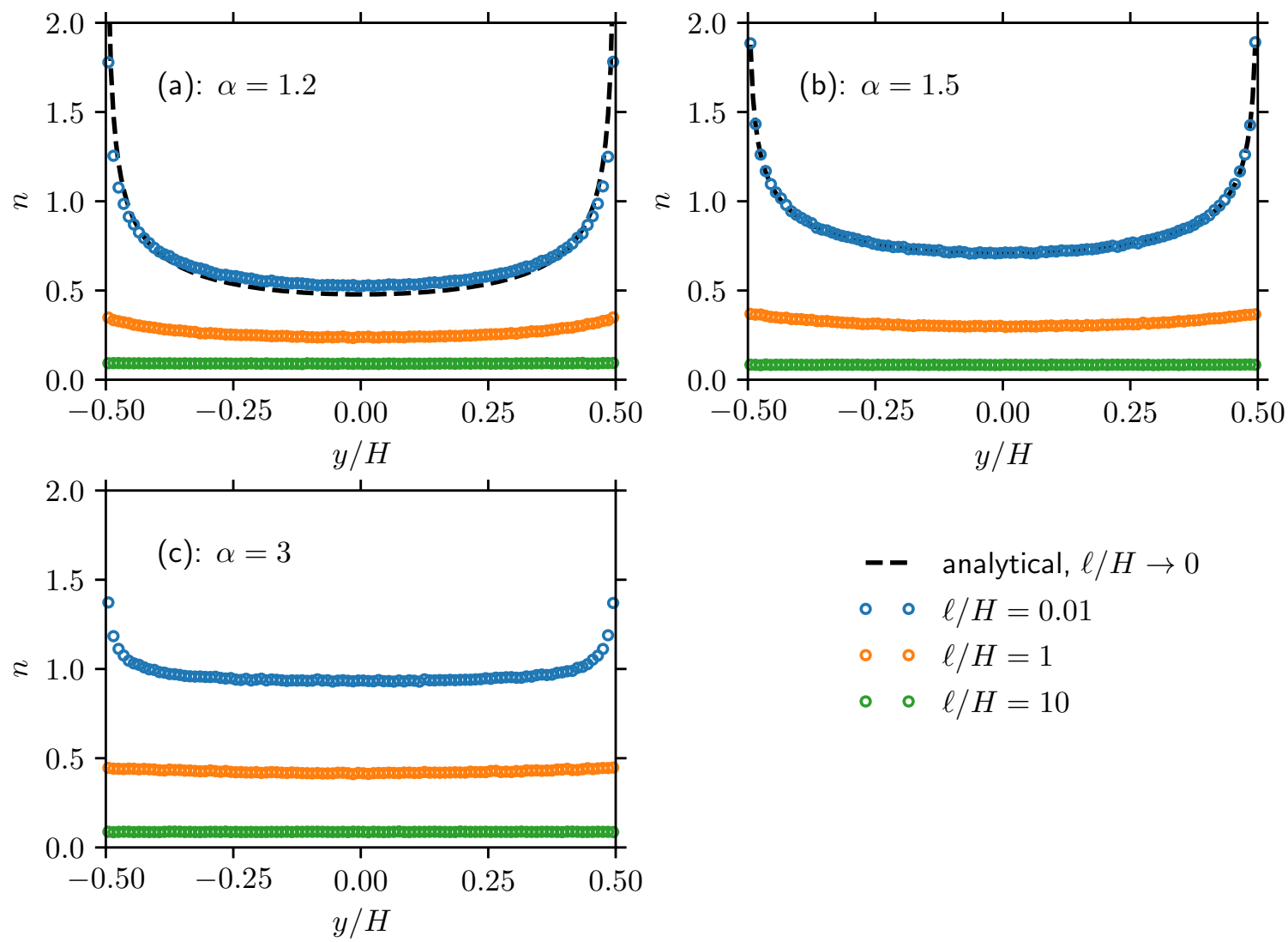

$$
\begin{array}{ll}
\text { - } & \text { analytical, } \ell / H \rightarrow 0 \\
\circ & \circ \quad \ell / H=0.01 \\
\circ & \circ \quad \ell / H=1 \\
\circ & \circ \quad \ell / H=10
\end{array}
$$

Figure 2. Simulated bulk density profile $n$ of swimmers with $(a) \alpha=1.2$, (b) $\alpha=1.5$ and $(c) \alpha=3.0$ in a 1D channel. The strong confinement limit of $(a-c)$ and the weak confinement limit of $(a-b)$ agree with the analysis in Sections 4 and 5 In contrast to $(a-b),(c)$ reveals convergence to the Gaussian limit with a constant bulk density and a thin boundary layer for $\ell / H \ll 1$, due to the finite variance of Pareto distribution with $\alpha=3>2$. This is consistent with the RTPs results reported in Ref. [58]. In all subfigures $(a-c)$, when normalizing the densities, only swimmers in the bulk, but not on the wall, are included. The swimmers accumulated on the walls are not shown here but they give rise to the forces on the wall shown in Figure 4 (a).

of swimmers are stuck on the wall. Hence, the total bulk fraction shown on the plots decreases from blue $(\ell / H=0.01)$ to orange $(\ell / H=1)$ to green $(\ell / H=10)$ curves.

In $2 \mathrm{D}$, the analysis remains true in the same asymptotic limit, with the slight modification that the swimmer orientation now ranges in $[0,2 \pi)$. At strong confinement, this leads to an $O\left((H / \ell)^{2}\right)$ correction to the average channel crossing time. The polar order distribution on the wall is modified by a prefactor of $\frac{1}{\pi} \int_{0}^{\pi} \sin (\theta) d \theta=2 / \pi$. The force exerted on a wall of the channel is therefore

$$
F_{\text {wall }}=\frac{C_{d}}{2}\left(1-\frac{H}{\ell}\right) N \zeta U_{0}
$$

where $N$ is the number of swimmers confined inside the channel, $\zeta U_{0}$ is their swim force, and $C_{d}=1$ for $1 \mathrm{D}$ and $C_{d}=2 / \pi$ for $2 \mathrm{D}$. As show in Figures 2 and 3 , the profiles for 

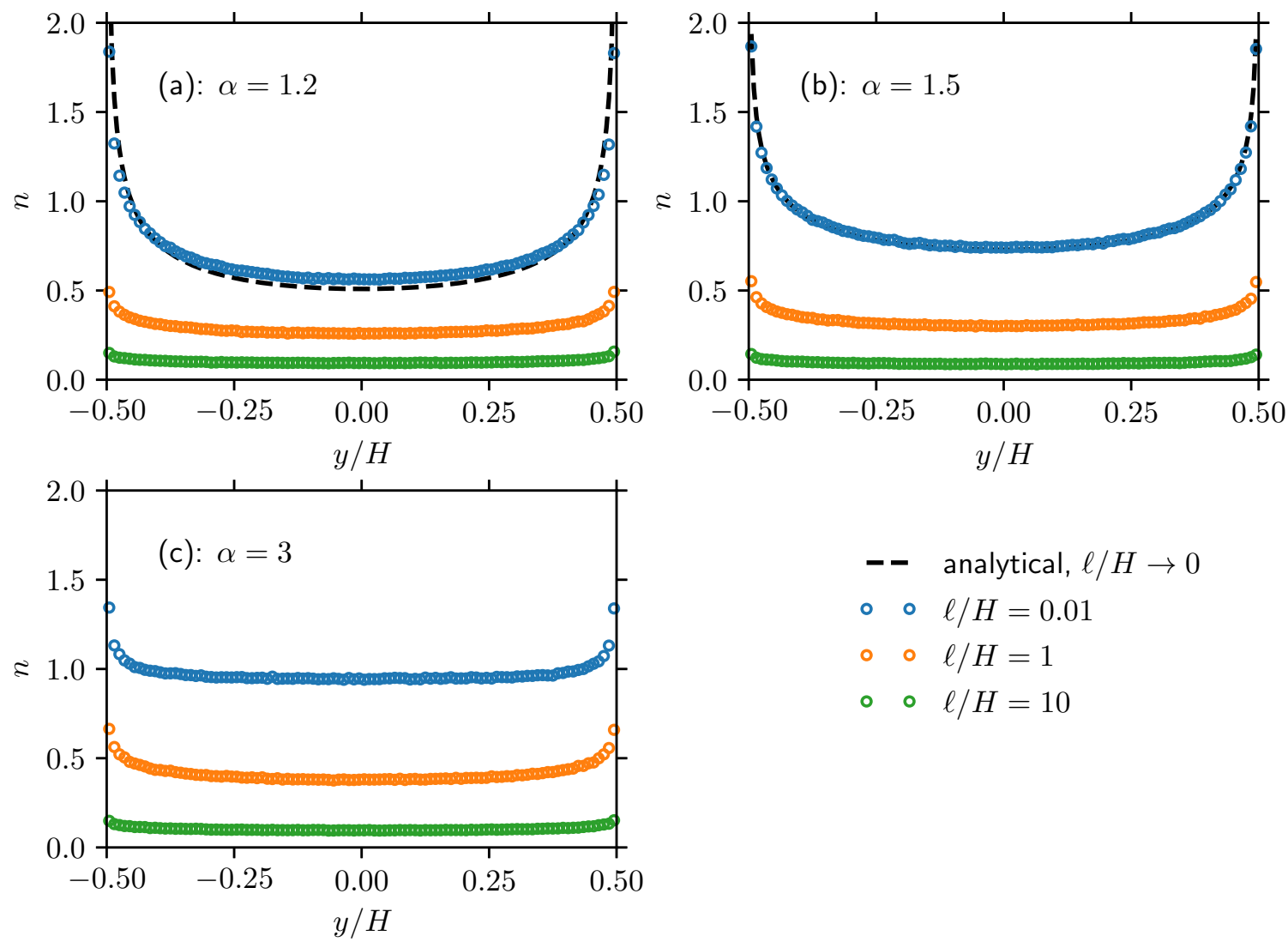

$$
\begin{array}{ll}
\text { - } & \text { analytical, } \ell / H \rightarrow 0 \\
\circ & \circ \quad \ell / H=0.01 \\
\circ & \circ \quad \ell / H=1 \\
\circ & \circ \quad \ell / H=10
\end{array}
$$

Figure 3. Simulated bulk density profile $n$ of swimmers with $(a) \alpha=1.2(b) \alpha=1.5$ and $(c) \alpha=3.0$ across a 2D channel. The weak confinement limit of $(c)$ converges to the CLT prediction of constant in the bulk, proving that the U-shape density profile in $(c)$ for intermediate $\ell / H$ is only a boundary layer effect [58], while in $(a-b)$ it is a bulk property of the superdiffusive behavior. When normalizing the densities, only swimmers in the bulk but not on the wall are included. The swimmers accumulated on the walls are not shown here but they give rise to the forces on the wall shown in Figure田(b).

$\ell / H=10$ have almost converged to uniform density distributions, confirming the above analysis. Each simulation curve integrated over the domain gives the corresponding bulk number of swimmers, not including the singular accumulation on the walls. As shown in Figure 4 (a), the force asymptotes represented by equation (13) agree well with the results of our particle simulations for large $\ell / H$. The same figure illustrates how the singular accumulation at the walls, quantified by $F_{\text {wall }}$, varies with $\ell / H$.

\section{Limit of weak confinement: fractional diffusion}

When $\ell / H \ll 1$, we expect that the swimmers in the bulk do not 'see' the boundary of the domain easily, and so can be modeled by the same equations as swimmers in free space, but with boundary conditions. In this section, we show this gives rise to 


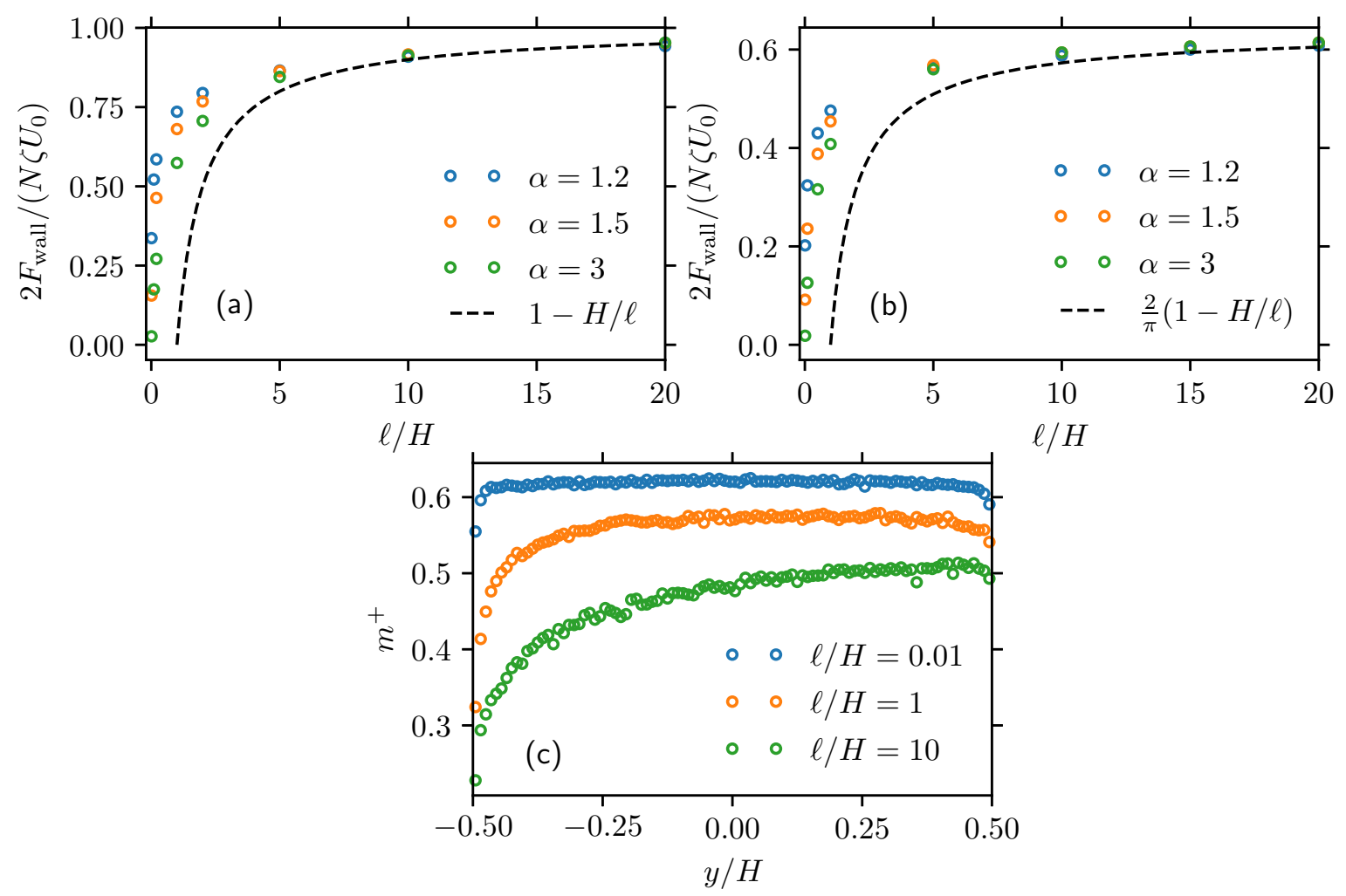

Figure 4. Forces of swimmers with $\alpha \in\{1.2,1.5,3\}$ exerted on the wall in (a) 1D and (b) 2D channels. The asymptotes are from the strong confinement limit analysis in Section 4 which is expected to be more accurate as $\ell / H$ becomes larger. (c) Distribution of the polarization $m^{+}$[see equation (44)] of 2D Lévy swimmers with $\alpha=1.2$ pointing towards the upper wall.

a fractional diffusion equation involving the Riesz definition of fractional derivatives in finite domains together with a no-flux boundary condition recently introduced by Kelly et al. [70] and Baeumer et al. [71].

In this limit, at times $t$ much larger than $\tau_{m}$, the system reaches a superdiffusive regime. This permits a multiscale analysis using a Hilbert expansion technique [72] to coarse-grain over the short time $\tau$ of ballistic motion. To prepare for this analysis, the kinetic equation (3) can be cast in Fourier space as

$$
\left(\frac{\partial}{\partial \tau}+\frac{\partial}{\partial t}+U_{0} \boldsymbol{q} \cdot(i \boldsymbol{\xi})\right) \hat{P}(\boldsymbol{\xi}, t, \tau, \boldsymbol{q})=-\beta(\tau) \hat{P}(\boldsymbol{\xi}, t, \tau, \boldsymbol{q}) ;
$$

here, we define the Fourier and inverse Fourier transforms, respectively, as

$$
\begin{aligned}
\mathcal{F}[u](\boldsymbol{\xi}) & =\frac{1}{(2 \pi)^{d / 2}} \int e^{-i \boldsymbol{r} \cdot \boldsymbol{\xi}} u(\boldsymbol{r}) d \boldsymbol{r}, \\
\mathcal{F}^{-1}[\hat{u}](\boldsymbol{r}) & =\frac{1}{(2 \pi)^{d / 2}} \int e^{i \boldsymbol{r} \cdot \boldsymbol{\xi}} \hat{u}(\boldsymbol{\xi}) d \boldsymbol{\xi},
\end{aligned}
$$


with the shorthand $\hat{u}=\mathcal{F}[u]$. We then integrate equation (14) over $\tau$ for the reduced probability density

$$
\hat{p}(\boldsymbol{\xi}, t, \boldsymbol{q})=\int_{0}^{t} \hat{P}(\boldsymbol{\xi}, t, \tau, \boldsymbol{q}) d \tau .
$$

In Fourier space, this leads (see equation (14) of Ref. [60]) to

$$
\left(\frac{\partial}{\partial t}+U_{0} \boldsymbol{q} \cdot(i \boldsymbol{\xi})\right) \hat{p}(\boldsymbol{\xi}, t, \boldsymbol{q})=(\mathcal{T}-\mathcal{I}) \int_{0}^{t} \beta(\tau) \hat{P} d \tau
$$

where $\mathcal{I}$ is the identity operator and $\mathcal{T}[f(\boldsymbol{q})]=\int_{S} f\left(\boldsymbol{q}^{\prime}\right) d \boldsymbol{q}^{\prime}$ is the turn angle operator, with $S$ denoting the unit sphere in $\mathbb{R}^{d}$.

Our goal is to derive the governing equation for the number density of swimmers

$$
n(\boldsymbol{r}, t):=\int_{S} p(\boldsymbol{r}, t, \boldsymbol{q}) d \boldsymbol{q} .
$$

The conservation of number density is expressed by

$$
\frac{\partial n}{\partial t}+\nabla \cdot j=0
$$

where $\boldsymbol{j}$ is the flux, which is defined as

$$
\boldsymbol{j}(\boldsymbol{r}, t):=\int_{S} U_{0} \boldsymbol{q} p(\boldsymbol{r}, t, \boldsymbol{q}) d \boldsymbol{q} .
$$

\subsection{Derivation of fractional-order flux via Hilbert Expansion}

Refs. [59, 60, 66] show that by assuming a superdiffusive power-law scaling for the leading order equation obtained in the Hilbert expansion analysis of equation (18), a fractional-order governing equation can be derived for the number density $n$ in free space. We summarize their derivation below, with the modification that the equations are represented in Fourier space. By expressing the derivations of Estrada-Rodriguez et al. [60] in Fourier space, their formal expansions in powers of differential operators become expansions in powers of scalars, which are well-defined. In turn, this justifies the definition of the specific fractional-order gradient operator in their final results. This operator was introduced by Meerschaert et al. [73] as

$$
\mathcal{F}\left[\nabla^{\alpha-1} u\right](\boldsymbol{\xi})=\left[\int_{S} \boldsymbol{q}(i \boldsymbol{\xi} \cdot \boldsymbol{q})^{\alpha-1} d \boldsymbol{q}\right] \hat{u}(\boldsymbol{\xi})
$$

Clarification of the specific fractional-order gradient operator is critical, as there are several notions of gradient in fractional calculus that are not equivalent [74, 75].

Starting from equation (18), Estrada-Rodriguez et al. [60] showed $p(\boldsymbol{r}, t, \boldsymbol{q})$ can be formally expanded in terms of $\boldsymbol{q} \cdot \nabla$, which is rigorous in Fourier space since $\boldsymbol{q} \cdot \nabla$ transforms into the scalar $\boldsymbol{q} \cdot(i \boldsymbol{\xi})$. The leading order of their expansion led to equation 
(46) in their article, which in Fourier space, and in the absense of external source terms, is represented as

$$
\int_{S} \boldsymbol{q} \frac{(\alpha-1)}{\tau_{0}} \boldsymbol{q} \cdot \hat{\boldsymbol{j}} d \boldsymbol{q}=\int_{S} \boldsymbol{q} \tau_{0}^{\alpha-2}(1-\alpha)^{2} \Gamma(1-\alpha) U_{0}^{\alpha}(\boldsymbol{q} \cdot(i \boldsymbol{\xi}))^{\alpha-1} \hat{n} d \boldsymbol{q} .
$$

In our case, this expression is simplified due to the uniform distribution of orientation angle after tumbling events. Estrada-Rodriguez et al. [60] then show that

$$
\int_{S} \boldsymbol{q} \frac{(\alpha-1)}{\tau_{0}} \boldsymbol{q} \cdot \hat{\boldsymbol{j}} d \boldsymbol{q}=\frac{(\alpha-1)}{\tau_{0}} \hat{\boldsymbol{j}}
$$

As a result of the last two equations, the Fourier transform of the flux $\hat{j}$ can be related to the Fourier transform of the number density $\hat{n}$,

$$
\hat{\boldsymbol{j}}=\frac{\pi(\alpha-1) \tau_{0}^{\alpha-1} U_{0}^{\alpha}}{\sin (\pi \alpha) \Gamma(\alpha)} \int_{S} \boldsymbol{q}(\boldsymbol{q} \cdot i \boldsymbol{\xi})^{\alpha-1} \hat{n} d \boldsymbol{q}
$$

This coincides with the definition of the fractional gradient (22), and when transformed to real space gives

$$
\boldsymbol{j}=\frac{\pi(\alpha-1) \tau_{0}^{\alpha-1} U_{0}^{\alpha}}{\sin (\pi \alpha) \Gamma(\alpha)} \nabla^{\alpha-1} n
$$

Inserting the flux expression into the conservation equation (20), and using the property [73]

$$
\nabla \cdot \nabla^{\alpha-1}=-(-\Delta)^{\alpha / 2}
$$

this finally leads to

$$
\frac{\partial n}{\partial t}-C_{\alpha}(-\Delta)^{\alpha / 2} n=0,
$$

where $C_{\alpha}=\pi(\alpha-1) \tau_{0}^{\alpha-1} U_{0}^{\alpha} /[\sin (\pi \alpha) \Gamma(\alpha)]$ and the fractional Laplacian is defined as

$$
-(\Delta)^{\alpha / 2} n(\boldsymbol{r})=\frac{2^{\alpha} \Gamma\left(\frac{\alpha}{2}+\frac{d}{2}\right)}{\pi^{d / 2}|\Gamma(-\alpha / 2)|} \int_{\mathbb{R}^{d}} \frac{n(\boldsymbol{r})-n\left(\boldsymbol{r}^{\prime}\right)}{\left|\boldsymbol{r}-\boldsymbol{r}^{\prime}\right|^{d+\alpha}} d \boldsymbol{r}^{\prime}
$$

It is possible to write

$$
-(-\Delta)^{\alpha / 2} n=\Delta I_{2-\alpha} n
$$

where $I_{2-\alpha}$ is a Riesz potential of order $2-\alpha$ applied to $n$, the inverse Fourier transform of which is $|\boldsymbol{\xi}|^{-(2-\alpha)} \hat{n}$. As a result, one can write equation (28) as

$$
\frac{\partial n(\boldsymbol{r}, t)}{\partial t}=-C_{\alpha} \Delta \int_{\mathbb{R}^{d}} \frac{n\left(\boldsymbol{r}^{\prime}, t\right)}{\left|\boldsymbol{r}-\boldsymbol{r}^{\prime}\right|^{1-(2-\alpha)}} d \boldsymbol{r}^{\prime}
$$

It is possible then to restrict integration of $\boldsymbol{r}^{\prime}$ to the channel. We take this step in the following section after considering the steady state of equation (28).

We remark here on equation (28): It is known that this fractional diffusion governs the probability distribution of a Lévy flight process in free space, with the solution exhibiting unbounded support due to the unbounded jumps inherent to the flight paths. 
Nevertheless, it has been derived as a first-order governing equation for the Lévy walk model that describes non-interacting active Lévy swimmers. Zaburdaev et al. [23] has demonstrated that the bulk density profile of Lévy walkers in free space is approximated by a Lévy stable distribution supported between two ballistic peaks that propagate with constant velocity. This result is consistent with the derivation of equation (28) as a leading-order equation for the distribution of Lévy walkers. Below, we carefully show how boundary conditions can be utilized to constrain the solution of equation (28) to describe the properties of the active Lévy swimmers introduced in Section 2, We also note that a Hilbert expansion analysis of normal run-and-tumble particles in 1D results in a telegraph equation [72, 76], which yields a propagating front with finite speed due to a second order time derivative term. It is possible that a more careful multiscale analysis for Lévy walks, which includes higher order effects, may yield an additional hyperbolic term in equation (28) to properly characterize the ballistic front.

\subsection{Zero exterior condition and no-flux boundary condition for the channel geometry}

In the previous section, we reviewed that a fractional diffusion equation governs the number density $n$. Here, we clarify the boundary conditions required to close the description of the active Lévy swimmers confined in the channel. Due to their discontinuous-in-time paths, boundary conditions for Lévy processes raise complicated issues, often requiring exterior conditions for their governing equations instead of standard boundary conditions [77].

According to the hard-wall interactions inherent in our model of active Lévy swimmers described in Section 2, particles cannot pass through boundaries of the channel, which manifests in two properties. First, the density vanishes,

$$
P(\boldsymbol{r}, t, \tau, \boldsymbol{q})=0,
$$

for $\boldsymbol{r}$ outside of the channel, which implies that

$$
n(\boldsymbol{r}, t)=0 .
$$

Second, the flux vanishes,

$$
\boldsymbol{j}(\boldsymbol{r}, t)=0,
$$

when $\boldsymbol{r}$ lies on the channel wall. For a classical diffusion equation, only the second condition is required, as it provides a Neumann boundary condition which determines the solution up to a constant. However, the fractional Laplacian (29), due to its nonlocal nature, requires an exterior condition represented by equation (33) to be welldefined [77]. Below, we will use conditions (33) and (34) in distinct ways to derive the steady state solution to equation (28).

\subsection{Steady state in $1 D$}

In 1D, the fractional Laplacian in equation (28) reduces to the the Riesz (or the Riesz-

Feller) fractional derivative in one dimension [70]. This operator can be written for 
$1<\alpha<2$ as

$$
\frac{\partial^{\alpha} n(y)}{\partial|y|^{\alpha}}=\frac{C_{\alpha}}{\Gamma(2-\alpha)} \frac{d^{2}}{d y^{2}} \int_{-\infty}^{\infty} \frac{n(x)}{|y-x|^{\alpha-1}} d x .
$$

Using the exterior condition (34), which in 1D implies that $n(y)=0$ for $y \notin$ $[-H / 2, H / 2]$, this reduces to

$$
\frac{\partial^{\alpha} n(y)}{\partial|y|^{\alpha}}=\frac{C_{\alpha}}{\Gamma(2-\alpha)} \frac{d^{2}}{d y^{2}} \int_{-H / 2}^{H / 2} \frac{n(x)}{|y-x|^{\alpha-1}} d x .
$$

Therefore, the steady state of equation (28) can be written as

$$
0=\frac{\partial^{\alpha} n(y)}{\partial|y|^{\alpha}}=\frac{C_{\alpha}}{\Gamma(2-\alpha)} \frac{d^{2}}{d y^{2}} \int_{-H / 2}^{H / 2} \frac{n(x)}{|y-x|^{\alpha-1}} d x .
$$

No-flux boundary conditions for the steady state equation (37) were derived by Kelly et al. [70]. They introduced the Riemann-Liouville flux, which in the symmetric case is given by

$$
j_{R L}=\frac{C_{\alpha}}{\Gamma(2-\alpha)} \frac{d}{d y} \int_{-H / 2}^{H / 2} \frac{n(x)}{|y-x|^{\alpha-1}} d x .
$$

This allowed them to write the steady state equation (37) in conservation form,

$$
0=\frac{d j_{R L}}{d y}=\frac{d}{d y}\left(\frac{C_{\alpha}}{\Gamma(2-\alpha)} \frac{d}{d y} \int_{-H / 2}^{H / 2} \frac{n(x)}{|y-x|^{\alpha-1}} d x\right) .
$$

Comparing this equation to equation (20) shows that $j_{R L}$ can be identified with $\boldsymbol{j}$ in $1 \mathrm{D}$, so that the no-flux boundary condition (34) implies $j_{R L}=0$ for $y= \pm H / 2$. Kelly et al. [70] derived a general solution to equation (37), which under the no-flux condition reduces to

$$
n(y)=C_{0}\left(\frac{1}{4}-\frac{y^{2}}{H^{2}}\right)^{\alpha / 2-1}
$$

where $C_{0}$ is a normalization constant determined by conservation of total number of swimmers.

As shown in Figure 2(a-b), the analytical solution agrees well with our simulation data in the weak confinement limit $(\ell / H \ll 1)$. In plotting Figures 2 and $3(\mathrm{a}-\mathrm{b})$, the analytic curve is normalized to have the same number of total swimmers as that of the bulk from the simulations. The fraction of swimmers accumulated on the wall push against the wall with a constant force, giving rise to the force measured in Figure 4 (a).

We remark that, in 1D, equation (28) with the boundary conditions (33) and (34) also governs the evolution of the density of $\alpha$-stable Lévy flights with the 'stopping' boundary condition studied by Dybiec et al. [61]. This condition is defined by the property that a flier which attempts to leave the interval is stopped near the corresponding endpoint until another jump is drawn from the $\alpha$-stable distribution that moves it back into the bulk. In this context, Denisov et al. [78] derived a steadystate solution that agrees with equation (37). However, the prescription of 'stopping' 
boundary conditions for Lévy flights in higher dimensions is more subtle and is not expected to agree with the sliding no-flux boundary conditions prescribed in our active Lévy swimmer model. We also remark that the shape of our steady state solution is similar to that observed for reflected fractional Brownian motion [40].

\subsection{Steady state in $2 D$}

In $2 \mathrm{D}$, we expect the steady state of the number density to be independent of the longitudinal coordinate $x$, i.e. $n(x, y)=n(y)$; see Figure 1. In this case, we show that the fractional Laplacian of $n(x, y)$ in equation (28) reduces to the one-dimensional Riesz derivative in $y$. In the classical case of integer order derivatives, this follows immediately from the expansion of the Laplacian in partial derivatives; in the fractional case, it requires a more involved proof.

In Fourier space, the fractional Laplacian can be represented as

$$
\begin{aligned}
\mathcal{F}_{x, y}\left[-(-\Delta)^{\alpha / 2} n(x, y)\right](\xi, \eta) & =\left|\xi^{2}+\eta^{2}\right|^{\alpha / 2} \mathcal{F}_{x, y}[n](\xi, \eta) \\
& =\left|\xi^{2}+\eta^{2}\right|^{\alpha / 2} \mathcal{F}_{x}\left[\mathcal{F}_{y}[n](\eta)\right](\xi, \eta) \\
& =\left|\xi^{2}+\eta^{2}\right|^{\alpha / 2} \mathcal{F}_{y}[n](\eta) \delta(\xi) .
\end{aligned}
$$

In the above $\mathcal{F}_{x}, \mathcal{F}_{y}$ and $\mathcal{F}_{x, y}$ denote the Fourier transform in $x, y$, and $(x, y)$, respectively; for an absolutely integrable function $u(x, y)$, these satisfy

$$
\mathcal{F}_{x, y}[u](\xi, \eta)=\mathcal{F}_{x}\left[\mathcal{F}_{y}[u](x, \eta)\right](\xi, \eta) .
$$

In equation (41), the frequency variable $\xi$ corresponds to transformation in $x$, while $\eta$ corresponds to transformation in $y$. The last equality in (41) uses the fact that $\mathcal{F}_{y}[n](x, \eta)=\mathcal{F}_{y}[n](\eta)$ does not depend on $x$, yielding a $\delta$-function Fourier transform in $x$. Taking the inverse Fourier transform, we obtain

$$
\begin{aligned}
-(-\Delta)^{\alpha / 2} n(x, y) & =\mathcal{F}_{\xi, \eta}^{-1}\left\{\left|\xi^{2}+\eta^{2}\right|^{\alpha / 2} \mathcal{F}_{y}[n] \delta(\eta)\right\} \\
& =\mathcal{F}_{\eta}^{-1}\left\{\left|\eta^{2}\right|^{\alpha / 2} \mathcal{F}_{y}[n](\eta)\right\} \\
& =\frac{\partial^{\alpha} n(y)}{\partial|y|^{\alpha}} .
\end{aligned}
$$

The Fourier representation of the Riesz derivative used to obtain the final line can be found in, e.g., Ref. [45]. Therefore, the steady-state equation for $n(y)$ in $2 \mathrm{D}$ is identical to equation (37) from the 1D case, with the same exterior and no-flux boundary conditions. It has the same solution (401). The solutions are compared with particle simulations in Figure 3. As in 1D, the density profiles shown only include swimmers in the bulk but not on the walls. A swimmer accumulated on the wall pushes against the upper/lower wall, and the normal component of the force $F \sin \theta^{ \pm}$is balanced by the wall. Averaged over swimmers for a finite time period this gives the force measured on the wall in Figure 4(b). Although we observe no local polar order in the bulk, the 
polar order of only those swimmers oriented towards the upper wall is not zero and not symmetric with respect to the center line of the channel

$$
m^{+}(y)=\int_{0}^{\pi} \cos \left(\theta^{+}\right) p\left(y, t=\infty, \theta^{+}\right) d \theta^{+} .
$$

This quantity is plotted in Figure 4(c).

\section{Comparisons between ABPs, normal RTPs and active Lévy swimmers}

Here we comment on the differences and similarities among the three species of active matter models. First, we compare ABPs and normal RTPs. It has been shown by a mean-field treatment of phenomenological models [3, 79, 80] that ABPs and RTPs behave similarly macroscopically, for example in motility-induced phase separation (MIPS). To further compare RTPs and ABPs, we prove that the stochastic dynamics of non-interacting ABPs leads to an exponential decay of their velocity autocorrelation function, coinciding with that of normal RTPs.

Without loss of generality we consider the 2D case. The stochastic dynamics of an ABP moving in $x y$ plane can be described by the Langevin equation

$$
\dot{\boldsymbol{v}}(t)=\eta(t) \boldsymbol{e}_{z} \times \boldsymbol{v}(t)
$$

with

$$
\begin{aligned}
\left\langle\eta\left(t_{1}\right) \eta\left(t_{2}\right) \cdots \eta\left(t_{2 m-1}\right)\right\rangle & =0 \\
\left\langle\eta\left(t_{1}\right) \eta\left(t_{2}\right) \cdots \eta\left(t_{2 m}\right)\right\rangle & =\sum_{<i, j>} \sigma^{2 m} \Pi_{<i, j>} \delta\left(t_{i}, t_{j}\right) \triangleq M_{2 m}
\end{aligned}
$$

where $\boldsymbol{v}$ is the velocity vector, $\eta(t)$ a Gaussian noise, and $\boldsymbol{e}_{z}$ the unit vector in $z$ direction. The decomposition of higher order moments into variance are due to the Wick theorem [81] for Gaussian noise.

By recursively expanding the velocity Langevin equation one can show that the Wick theorem leads to exponential decay of the velocity autocorrelations; see Appendix C for this derivation. Using that result, the MSD for non-interacting ABPs is [6, 82]

$$
\left\langle r^{2}(t)\right\rangle=v_{0}^{2}\left\langle\int_{0}^{t} d \tau \int_{0}^{t} d \tau^{\prime} e^{-\frac{\sigma^{2}}{2}\left|\tau-\tau^{\prime}\right|}\right\rangle=\frac{4 v_{0}^{2}}{\sigma^{2}}\left(t-\frac{2}{\sigma^{2}}\left(1-e^{-\frac{\sigma^{2}}{2} t}\right)\right),
$$

which at long times satisfies the scaling relation

$$
\left\langle r^{2}(t)\right\rangle \propto t \quad \text { as } \quad t \rightarrow \infty
$$

while the MSD for non-interacting Lévy swimmers scales as [23]

$$
\left\langle r^{2}(t)\right\rangle \propto t^{3-\alpha} \quad \text { as } \quad t \rightarrow \infty
$$


This reveals that ABPs exhibit behavior that is ballistic at short time scales, and normally diffusive at long time scales. This also holds for normal RTPs, as can be shown using Hilbert expansion analysis [68, 69]. In contrast, the Lévy swimmers exhibit superdiffusive propagation at long times. The Lévy swimmers also exhibit ballistic behavior at short time scales, but the ballistic front of the densities of ABP/RTP and Lévy swimmers are qualitatively different. The short persistent times of ABP/RTP yields ballistic fronts that decay rapidly, leading to a self-similar property of the density in time characterized by a length scale proportional to $t^{1 / 2}$. This is consistent with the MSD scaling given by equation (48). In contrast, the ballistic front of the density of Lévy swimmers, while decaying, persists to an extent that the density is not selfsimilar in time. However, the center of the density profile follows a self-similar scaling proportional to $t^{1 / \alpha}$ [23], which is distinct from the MSD scaling given by equation (49)).

Active Lévy swimmers share the discrete tumbling behavior as normal RTPs; neither involve translational diffusion. Our analysis and simulations show clearly a singular accumulation of active Lévy swimmers on the walls of the channel, similar to RTPs. However, most importantly, the correlation of Lévy swimmers in the bulk is qualitatively non-local and the density distributions converge to a non-uniform steadystate distribution inside the bulk at small $\ell / H$, which is well approximated by the steady-state solution (37). This novel aspect distinguishes active Lévy swimmers from both RTPs and ABPs.

\section{Conclusion and Discussions}

We introduce a model for active swimmers with Lévy statistics under confinement. The Lévy swimmers are characterized by the power-law tail of their run-time distribution $\psi(\tau)$, which is taken as the Pareto distribution, and a constant swim force and hence a constant velocity magnitude $U_{0}$ between tumbling events when not interacting with other objects. On the domain boundaries we assume the Lévy swimmers follow the Skorokhod sliding condition, similar to the ABP and RTP models. Our modification to previous multiscale analysis (Hilbert expansion) shows that a fractional diffusion equation still holds as the leading order description in the weak confinement regime, with the Riesz fractional derivative and no-flux boundary conditions. In this asymptotic limit of $\ell / H \rightarrow 0$ the Lévy swimmers show qualitative difference from the ABP and RTP models, with a non-uniform U-shaped bulk density distribution. This is distinctive from the accumulation boundary layer effect of ABPs and RTPs, which scales with activity and dimishes as $\ell / H$ decreases, eventually recovering a uniform bulk distribution. In the strong confinement limit neither the Lévy swimmers nor the RTPs have time to tumble in the bulk of the channel, which results in a uniform bulk density profile. The fraction of swimmers accumulated and the force exerted on the walls are shown to converge to an analytic asymptote. Our analysis agree with particle simulations of stochastic trajectories of the swimmers. Here, we show results for $1 \mathrm{D}$ and $2 \mathrm{D}$ only, but the analysis can be easily generalized to $3 \mathrm{D}$. 
One advantage of our Lévy swimmer model compared to Lévy flights or walks is that the only prescription of swim force and drag coefficient readily admits extensions to interacting swimmers with finite sizes. In our preliminary simulations we have observed motility-induced phase separation of Lévy swimmers interacting with the Weeks-Chandler-Andersen (WCA) potential [83] at high packing density and activity. In future work, we will investigate how the phase diagram and the universality class may change from ABPs and normal RTPs to Lévy swimmers. It is also interesting to compare the behaviors of Lévy swimmers with ABP/RTP in an external field.

Our study of active Lévy swimmers connects the physics of Lévy walks with recent developments in fractional calculus. This provides a mathematical foundation to study Lévy walks with interactions confined in more general geometries. The distinguishing properties of active Lévy swimmers, as compared to RTP and ABP models, show that they are a promising model for active matter in upstream swimming and transport in porous media.

\section{Acknowledgments}

We thank Z. He, S. Yip, H. Row, Z.G. Wang and C. Kjeldbjerg for insightful discussions. T.Z. is supported by the Cecil and Sally Drinkward Postdoc Fellowship. T.Z. and M.G. are grateful for the opportunity to hold discussions at the 2018 ICERM workshop "Fractional PDEs: Theory, Algorithms and Applications" at Brown University. J.F.B. is supported by NSF grant CBET 1803662.

M.G. is supported by the John von Neumann fellowship at Sandia National Laboratories. Sandia National Laboratories is a multimission laboratory managed and operated by National Technology and Engineering Solutions of Sandia, LLC., a wholly owned subsidiary of Honeywell International, Inc., for the U.S. Department of Energy's National Nuclear Security Administration under contract DE-NA0003525. The views expressed in the article do not necessarily represent the views of the U.S. Department of Energy or the United States Government. SAND number: SAND2021-2756 O.

\section{Appendix A. Details of particle simulations}

Our simulation data for Figures 2, 3, and 4 were obtained from the stochastic dynamics of the Lévy swimmers described in Section 2. At the beginning of each simulation, 100,000 swimmers are released from randomized initial positions within the channel, with uniformly random initial orientations. A run-time sampled from the type-II Pareto distribution $\psi(\tau)$ given by equation (1) is assigned to each swimmer. After a swimmer runs for this time, it is assigned a new orientation from a uniform angular distribution. In $1 \mathrm{D}$, this assigns probabilities of $1 / 2$ for both the left and right directions. In $2 \mathrm{D}, \theta$ is distributed in $[0,2 \pi)$. At the same time, a new run-time is sampled again from $\psi(\tau)$ and assigned to this swimmer. Between these tumbling events, a swimmer's trajectory is explicitly integrated with a timestep of $\Delta t=10^{-4} \tau_{m}$. When it hits the walls, the 
sliding no-flux boundary condition is imposed via a potential-free algorithm [84]. In 2D, a periodic boundary condition is imposed for the direction along the channel. Each simulation is run for $O\left(10^{2} \tau_{m}\right)$ to ensure sufficient convergence to the steady state, and statistics are measured by averaging over all swimmers for the last $O\left(10 \tau_{m}\right)$ time interval.

\section{Appendix B. Kinetic equations at steady state}

At steady state, explicit dependence on time and on the direction $x$ parallel to the channel disappears. Hence, in 2D the microscopic equations (3) - (9) reduce to

$$
\left\{\begin{aligned}
\left(\frac{\partial}{\partial \tau}+U_{0} \sin \theta \frac{\partial}{\partial y}\right) P(y, \theta, \tau) & =-\beta(\tau) P(y, \theta, \tau) \\
\frac{\partial \phi^{+}}{\partial \tau} & =-\beta(\tau) \phi^{+}+j_{b}^{+} \\
j_{b}^{+} & =U_{0} P\left(y=H / 2, \tau, \theta^{+}\right) \sin \theta^{+} \\
\phi^{+}\left(\tau=0, \theta^{+}\right) & =-P\left(y=H / 2, \tau=0, \theta^{-}\right) U_{0} \sin \theta^{-} \\
& =\int_{0}^{t} \beta(\tau) d \tau \frac{1}{2 \pi} \int_{0}^{\pi} d \theta^{\prime} \phi^{+} \\
& \approx \int_{0}^{\infty} \beta(\tau) d \tau \frac{1}{2 \pi} \int_{0}^{\pi} d \theta^{\prime} \phi^{+} .
\end{aligned}\right.
$$

In $1 \mathrm{D}$, the steady state equations are

$$
\left\{\begin{aligned}
\left(\frac{\partial}{\partial \tau}+U_{0} \frac{\partial}{\partial x}\right) P_{ \pm}(x, \tau) & =-\beta(\tau) P_{ \pm} \\
\int_{0}^{t \rightarrow \infty} \beta(\tau) d \tau P_{ \pm} & =P_{\mp}(x, \tau=0) \\
\frac{\partial \phi^{+}}{\partial \tau} & =-\beta(\tau) \phi^{+}+j_{b}^{+} \\
j_{b}^{+}(\tau) & =U_{0} P_{+}(x=H / 2, \tau) \\
\int_{0}^{\infty} d \tau \beta(\tau) \phi^{+} & =U_{0} P_{-}(x=H / 2, \tau=0) .
\end{aligned}\right.
$$

\section{Appendix C. Derivation of velocity autocorrelation function for ABPs}

Using a triple product identity and the fact that the particle moves in the $x y$ plane,

$$
\boldsymbol{e}_{z} \times\left(\boldsymbol{e}_{z} \times \boldsymbol{v}\right)=\left(\boldsymbol{e}_{z} \cdot \boldsymbol{v}\right) \boldsymbol{e}_{z}-\left(\boldsymbol{e}_{z} \cdot \boldsymbol{e}_{z}\right) \boldsymbol{v}=-\boldsymbol{v} .
$$

Therefore, we can recursively expand the velocity as

$$
\begin{aligned}
\boldsymbol{e}_{z} \times \boldsymbol{v}(t) & =\boldsymbol{e}_{z} \times\left(\boldsymbol{v}_{0}+\int_{0}^{t} d \tau \eta(\tau) \boldsymbol{e}_{z} \times \boldsymbol{v}(\tau)\right) \\
& =\boldsymbol{e}_{z} \times \boldsymbol{v}_{0}-\int_{0}^{t} d \tau \eta(\tau) \boldsymbol{v}(\tau)
\end{aligned}
$$


where $\boldsymbol{v}_{0}$ is the initial velocity. Hence

$$
\begin{aligned}
\langle\boldsymbol{v}(t)\rangle & =\boldsymbol{v}_{0}+\int_{0}^{t} d t_{1}\left\langle\eta\left(t_{1}\right) \boldsymbol{e}_{z} \times \boldsymbol{v}\left(t_{1}\right)\right\rangle \\
& =\boldsymbol{v}_{0}+\int_{0}^{t} d t_{1}\left(\left\langle\eta\left(t_{1}\right)\right\rangle \boldsymbol{e}_{z} \times \boldsymbol{v}_{0}-\int_{0}^{t_{1}} d t_{2}\left\langle\eta\left(t_{1}\right) \eta\left(t_{2}\right) \boldsymbol{v}\left(t_{2}\right)\right\rangle\right) \\
& =\boldsymbol{v}_{0}-\int_{0}^{t} d t_{1} \int_{0}^{t_{1}} d t_{2}\left\langle\eta\left(t_{1}\right) \eta\left(t_{2}\right) \boldsymbol{v}\left(t_{2}\right)\right\rangle \\
& =\boldsymbol{v}_{0}-\int_{0}^{t} d t_{1} \int_{0}^{t_{1}} d t_{2}\left\langle\eta\left(t_{1}\right) \eta\left(t_{2}\right)\right\rangle+\int_{0}^{t} \int_{0}^{t_{1}} \int_{0}^{t_{2}} \int_{0}^{t_{3}} \prod_{i=1}^{4} d t_{i}\left\langle\eta\left(t_{1}\right) \eta\left(t_{2}\right) \eta\left(t_{3}\right) \eta\left(t_{4}\right)\right\rangle-\ldots \\
& =\boldsymbol{v}_{0}\left(1-t \sigma^{2} / 2+\frac{t^{2}}{2} \sigma^{4} / 4\right)-\int \ldots\left\langle\ldots \boldsymbol{v}\left(t_{6}\right)\right\rangle \\
& =\boldsymbol{v}_{0} \sum_{n=0}^{\infty} \frac{1}{n !}\left(-t \sigma^{2} / 2\right)^{n} \\
& =\boldsymbol{v}_{0} e^{-t \sigma^{2} / 2} .
\end{aligned}
$$

The above derivation follows from applying the Wick theorem [81]. Due to the hierarchy of upper limits of integrations, only the leading term of the Wick summation survives. The evaluation of the integrals uses equation (46) and the fact that

$$
\delta(x-y)=\lim _{\tau \rightarrow 0} \begin{cases}1 / \tau & \text { if }|x-y|<\tau \\ 0 & \text { otherwise. }\end{cases}
$$

\section{References}

[1] J Elgeti, R G Winkler, and G Gompper. Physics of microswimmers - single particle motion and collective behavior: A review. Reports on Progress in Physics, 78(5): 056601, Apr 2015. doi: 10.1088/0034-4885/78/5/056601.

[2] Clemens Bechinger, Roberto Di Leonardo, Hartmut Löwen, Charles Reichhardt, Giorgio Volpe, and Giovanni Volpe. Active particles in complex and crowded environments. Rev. Mod. Phys., 88:045006, Nov 2016. doi: 10.1103/RevModPhys. 88.045006 .

[3] Michael E Cates and Julien Tailleur. Motility-induced phase separation. Annu. Rev. Condens. Matter Phys., 6(1):219-244, 2015.

[4] M. C. Marchetti, J. F. Joanny, S. Ramaswamy, T. B. Liverpool, J. Prost, Madan Rao, and R. Aditi Simha. Hydrodynamics of soft active matter. Rev. Mod. Phys., 85:1143-1189, Jul 2013. doi: 10.1103/RevModPhys.85.1143.

[5] Frank Jülicher, Stephan W Grill, and Guillaume Salbreux. Hydrodynamic theory of active matter. Reports on Progress in Physics, 81(7):076601, Jun 2018. doi: 10.1088/1361-6633/aab6bb. 
[6] Pawel Romanczuk, Markus Bär, Werner Ebeling, Benjamin Lindner, and Lutz Schimansky-Geier. Active Brownian particles. The European Physical Journal Special Topics, 202(1):1-162, 2012.

[7] Étienne Fodor, Cesare Nardini, Michael E Cates, Julien Tailleur, Paolo Visco, and Frédéric van Wijland. How far from equilibrium is active matter? Physical review letters, 117(3):038103, 2016.

[8] Étienne Fodor and M Cristina Marchetti. The statistical physics of active matter: From self-catalytic colloids to living cells. Physica A: Statistical Mechanics and its Applications, 504:106-120, 2018.

[9] Gandhimohan M Viswanathan, V Afanasyev, SV Buldyrev, EJ Murphy, PA Prince, and H Eugene Stanley. Lévy flight search patterns of wandering albatrosses. Nature, 381(6581):413-415, 1996.

[10] Andrew M Edwards, Richard A Phillips, Nicholas W Watkins, Mervyn P Freeman, Eugene J Murphy, Vsevolod Afanasyev, Sergey V Buldyrev, Marcos GE da Luz, Ernesto P Raposo, H Eugene Stanley, et al. Revisiting Lévy flight search patterns of wandering albatrosses, bumblebees and deer. Nature, 449(7165):1044-1048, 2007.

[11] Nuris Figueroa-Morales, Aramis Rivera, Rodrigo Soto, Anke Lindner, Ernesto Altshuler, and Éric Clément. E. coli "super-contaminates" narrow ducts fostered by broad run-time distribution. Science Advances, 6(11):eaay0155, 2020.

[12] Nuris Figueroa-Morales, Rodrigo Soto, Gaspard Junot, Thierry Darnige, Carine Douarche, Vincent A Martinez, Anke Lindner, and Eric Clément. 3D spatial exploration by E. coli echoes motor temporal variability. Physical Review X, 10 (2):021004, 2020.

[13] Haiyan Huo, Rui He, Rongjing Zhang, and Junhua Yuan. Swimming Escherichia coli explore the environment by Lévy walk. Applied and Environmental Microbiology, 2021.

[14] Ekaterina Korobkova, Thierry Emonet, Jose MG Vilar, Thomas S Shimizu, and Philippe Cluzel. From molecular noise to behavioural variability in a single bacterium. Nature, 428(6982):574-578, 2004.

[15] M Levandowsky, BS White, and FL Schuster. Random movements of soil amebas. Acta Protozoologica, 4(36), 1997.

[16] Elitsa Asenova, Hsin-Yu Lin, Eileen Fu, and Dan V Nicolau. Optimal fungal space searching algorithms. IEEE transactions on nanobioscience, 15(7):613-618, 2016.

[17] Tajie H Harris, Edward J Banigan, David A Christian, Christoph Konradt, Elia D Tait Wojno, Kazumi Norose, Emma H Wilson, Beena John, Wolfgang Weninger, Andrew D Luster, et al. Generalized Lévy walks and the role of chemokines in migration of effector CD8+ T cells. Nature, 486(7404):545-548, 2012.

[18] Marta C Gonzalez, Cesar A Hidalgo, and Albert-Laszlo Barabasi. Understanding individual human mobility patterns. Nature, 453(7196):779-782, 2008. 
[19] Dirk Brockmann, Lars Hufnagel, and Theo Geisel. The scaling laws of human travel. Nature, 439(7075):462-465, 2006.

[20] Dirk Brockmann and Dirk Helbing. The hidden geometry of complex, networkdriven contagion phenomena. Science, 342(6164):1337-1342, 2013.

[21] Michael F Shlesinger, Joseph Klafter, and Bruce J West. Lévy walks with applications to turbulence and chaos. Physica A: Statistical Mechanics and its Applications, 140(1-2):212-218, 1986.

[22] Michael F Shlesinger and Joseph Klafter. Lévy walks versus Lévy flights. In On growth and form, pages 279-283. Springer, 1986.

[23] V Zaburdaev, S Denisov, and J Klafter. Lévy walks. Reviews of Modern Physics, $87(2): 483,2015$.

[24] Kiyoshi Kanazawa, Tomohiko G Sano, Andrea Cairoli, and Adrian Baule. Loopy lévy flights enhance tracer diffusion in active suspensions. Nature, 579(7799):364367, 2020.

[25] Gandimohan M Viswanathan, Sergey V Buldyrev, Shlomo Havlin, MGE Da Luz, EP Raposo, and H Eugene Stanley. Optimizing the success of random searches. Nature, 401(6756):911-914, 1999.

[26] Michael A Lomholt, Koren Tal, Ralf Metzler, and Klafter Joseph. Lévy strategies in intermittent search processes are advantageous. Proceedings of the National Academy of Sciences, 105(32):11055-11059, 2008.

[27] Olivier Bénichou, Claude Loverdo, Michel Moreau, and Raphael Voituriez. Intermittent search strategies. Reviews of Modern Physics, 83(1):81, 2011.

[28] Gandhimohan M Viswanathan, Marcos GE Da Luz, Ernesto P Raposo, and $\mathrm{H}$ Eugene Stanley. The physics of foraging: an introduction to random searches and biological encounters. Cambridge University Press, 2011.

[29] Andy M Reynolds and Christopher J Rhodes. The Lévy flight paradigm: random search patterns and mechanisms. Ecology, 90(4):877-887, 2009.

[30] Nicolas E Humphries, Henri Weimerskirch, Nuno Queiroz, Emily J Southall, and David W Sims. Foraging success of biological Lévy flights recorded in situ. Proceedings of the National Academy of Sciences, 109(19):7169-7174, 2012.

[31] Surya G Nurzaman, Yoshio Matsumoto, Yutaka Nakamura, Satoshi Koizumi, and Hiroshi Ishiguro. Yuragi-based adaptive searching behavior in mobile robot: From bacterial chemotaxis to Lévy walk. In 2008 IEEE International Conference on Robotics and Biomimetics, pages 806-811. IEEE, 2009.

[32] Donny K Sutantyo, Serge Kernbach, Paul Levi, and Valentin A Nepomnyashchikh. Multi-robot searching algorithm using Lévy flight and artificial potential field. In 2010 IEEE Safety Security and Rescue Robotics, pages 1-6. IEEE, 2010.

[33] Surya G Nurzaman, Yoshio Matsumoto, Yutaka Nakamura, Kazumichi Shirai, Satoshi Koizumi, and Hiroshi Ishiguro. An adaptive switching behavior between 
Lévy and Brownian random search in a mobile robot based on biological fluctuation. In 2010 IEEE/RSJ International Conference on Intelligent Robots and Systems, pages 1927-1934. IEEE, 2010.

[34] Gissell Estrada-Rodriguez and Heiko Gimperlein. Interacting particles with Lévy strategies: limits of transport equations for swarm robotic systems. SIAM Journal on Applied Mathematics, 80(1):476-498, 2020.

[35] Étienne Fodor, Hisao Hayakawa, Julien Tailleur, and Frédéric van Wijland. Nongaussian noise without memory in active matter. Physical Review E, 98(6):062610, 2018.

[36] Andrea Cairoli and Chiu Fan Lee. Active l\'evy matter: Anomalous diffusion, hydrodynamics and linear stability. arXiv preprint arXiv:1904.08326, 2019.

[37] Laurent Decreusefond et al. Stochastic analysis of the fractional Brownian motion. Potential Analysis, 10(2):177-214, 1999.

[38] Francesca Biagini, Yaozhong Hu, Bernt Øksendal, and Tusheng Zhang. Stochastic calculus for fractional Brownian motion and applications. Springer Science \& Business Media, 2008.

[39] Thomas Vojta, Sarah Skinner, and Ralf Metzler. Probability density of the fractional Langevin equation with reflecting walls. Physical Review E, 100(4): 042142, 2019.

[40] Thomas Vojta, Samuel Halladay, Sarah Skinner, Skirmantas Janušonis, Tobias Guggenberger, and Ralf Metzler. Reflected fractional Brownian motion in one and higher dimensions. Physical Review E, 102(3):032108, 2020.

[41] Edmundo Capelas De Oliveira and José António Tenreiro Machado. A review of definitions for fractional derivatives and integral. Mathematical Problems in Engineering, 2014, 2014.

[42] G Sales Teodoro, JA Tenreiro Machado, and E Capelas De Oliveira. A review of definitions of fractional derivatives and other operators. Journal of Computational Physics, 388:195-208, 2019.

[43] Paul Lévy. Théorie de l'addition des variables aléatoires. Gauthier-Villars, 1954.

[44] BV Gnedenko, AN Kolmogorov, BV Gnedenko, and AN Kolmogorov. Limit distributions for sums of independent random variables. Am. J. Math, 105, 1954.

[45] Mark M Meerschaert and Alla Sikorskii. Stochastic models for fractional calculus, volume 43. Walter de Gruyter GmbH \& Co KG, 2019.

[46] Mark M Meerschaert and Hans-Peter Scheffler. Limit distributions for sums of independent random vectors: Heavy tails in theory and practice, volume 321. John Wiley \& Sons, 2001.

[47] John P Nolan. Univariate Stable Distributions: Models for Heavy Tailed Data. Springer Nature, 2020.

[48] Elliott W Montroll and George H Weiss. Random walks on lattices II. Journal of Mathematical Physics, 6(2):167-181, 1965. 
[49] Andrey Sokolov, Mario M. Apodaca, Bartosz A. Grzybowski, and Igor S. Aranson. Swimming bacteria power microscopic gears. Proceedings of the National Academy of Sciences, 107(3):969-974, 2010. ISSN 0027-8424. doi: 10.1073/pnas.0913015107.

[50] Raffaele Di Giacomo, Sebastian Krödel, Bruno Maresca, Patrizia Benzoni, Roberto Rusconi, Roman Stocker, and Chiara Daraio. Deployable micro-traps to sequester motile bacteria. Scientific Reports, 7(1):1-8, 2017.

[51] Lothar Wondraczek, Georg Pohnert, Felix H Schacher, Angela Köhler, Michael Gottschaldt, Ulrich S Schubert, Kirsten Küsel, and Axel A Brakhage. Artificial microbial arenas: Materials for observing and manipulating microbial consortia. Advanced Materials, 31(24):1900284, 2019.

[52] Sören B Gutekunst, Katharina Siemsen, Steven Huth, Anneke Möhring, Britta Hesseler, Michael Timmermann, Ingo Paulowicz, Yogendra Kumar Mishra, Leonard Siebert, Rainer Adelung, et al. 3D hydrogels containing interconnected microchannels of subcellular size for capturing human pathogenic Acanthamoeba Castellanii. ACS Biomaterials Science \& Engineering, 5(4):1784-1792, 2019.

[53] Zhiwei Peng and John F Brady. Upstream swimming and Taylor dispersion of active Brownian particles. Physical Review Fluids, 5(7):073102, 2020.

[54] Abdallah Daddi-Moussa-Ider, Maciej Lisicki, and Arnold JTM Mathijssen. Tuning the upstream swimming of microrobots by shape and cargo size. Physical Review Applied, 14(2):024071, 2020.

[55] Fernando Soto, Jie Wang, Rajib Ahmed, and Utkan Demirci. Medical micro/nanorobots in precision medicine. Advanced Science, 7(21):2002203, 2020.

[56] Camilla M Kjeldbjerg and John F Brady. Theory for the casimir effect and the partitioning of active matter. Soft Matter, 17(3):523-530, 2021.

[57] Luca Angelani. Confined run-and-tumble swimmers in one dimension. Journal of Physics A: Mathematical and Theoretical, 50(32):325601, 2017.

[58] Barath Ezhilan, Roberto Alonso-Matilla, and David Saintillan. On the distribution and swim pressure of run-and-tumble particles in confinement. Journal of Fluid Mechanics, 781:R4, 2015. doi: 10.1017/jfm.2015.520.

[59] Benoît Perthame, Weiran Sun, and Min Tang. The fractional diffusion limit of a kinetic model with biochemical pathway. Zeitschrift für angewandte Mathematik und Physik, 69(3):1-15, 2018.

[60] Gissell Estrada-Rodriguez, Heiko Gimperlein, and Kevin J Painter. Fractional Patlak-Keller-Segel equations for chemotactic superdiffusion. SIAM Journal on Applied Mathematics, 78(2):1155-1173, 2018.

[61] Bartłomiej Dybiec, Ewa Gudowska-Nowak, Eli Barkai, and Alexander A Dubkov. Lévy flights versus lévy walks in bounded domains. Physical Review E, 95(5): $052102,2017$.

[62] Kenneth S Lomax. Business failures: Another example of the analysis of failure 
data. Journal of the American Statistical Association, 49(268):847-852, 1954. doi: 10.1080/01621459.1954.10501239.

[63] Tony Albers and Günter Radons. Exact results for the nonergodicity of $d$ dimensional generalized Lévy walks. Physical review letters, 120(10):104501, 2018.

[64] Anatoliy V Skorokhod. Stochastic equations for diffusion processes in a bounded region. Theory of Probability \& Its Applications, 6(3):264-274, 1961.

[65] Paul Dupuis and Kavita Ramanan. Convex duality and the Skorokhod problem I. Probability Theory and Related Fields, 115(2):153-195, 1999.

[66] Martin Frank and Weiran Sun. Fractional diffusion limits of non-classical transport equations. arXiv preprint arXiv:160\%.04028, 2016.

[67] Wolgang Alt. Biased random walk models for chemotaxis and related diffusion approximations. Journal of mathematical biology, 9(2):147-177, 1980.

[68] Hans G Othmer and Thomas Hillen. The diffusion limit of transport equations derived from velocity-jump processes. SIAM Journal on Applied Mathematics, 61 (3):751-775, 2000.

[69] Ramón G Plaza. Derivation of a bacterial nutrient-taxis system with doubly degenerate cross-diffusion as the parabolic limit of a velocity-jump process. Journal of mathematical biology, 78(6):1681-1711, 2019.

[70] James F Kelly, Harish Sankaranarayanan, and Mark M Meerschaert. Boundary conditions for two-sided fractional diffusion. Journal of Computational Physics, 376:1089-1107, 2019.

[71] Boris Baeumer, Mihály Kovács, Mark M Meerschaert, and Harish Sankaranarayanan. Boundary conditions for fractional diffusion. Journal of Computational and Applied Mathematics, 339:414-430, 2018.

[72] Richard S Ellis. Chapman-Enskog-Hilbert expansion for a Markovian model of the Boltzmann equation. Communications on Pure and Applied Mathematics, 26(3): 327-359, 1973.

[73] Mark M Meerschaert, Jeff Mortensen, and Stephen W Wheatcraft. Fractional vector calculus for fractional advection-dispersion. Physica A: Statistical Mechanics and its Applications, 367:181-190, 2006.

[74] Marta D'Elia, Mamikon Gulian, Hayley Olson, and George Em Karniadakis. A unified theory of fractional, nonlocal, and weighted nonlocal vector calculus. arXiv preprint arXiv:2005.07686, 2020.

[75] Miroslav Šilhavỳ. Fractional vector analysis based on invariance requirements (critique of coordinate approaches). Continuum Mechanics and Thermodynamics, 32(1):207-228, 2020.

[76] HP McKean Jr. Chapman-enskog-hilbert expansion for a class of solutions of the telegraph equation. Journal of Mathematical Physics, 8(3):547-552, 1967. 
[77] Anna Lischke, Guofei Pang, Mamikon Gulian, Fangying Song, Christian Glusa, Xiaoning Zheng, Zhiping Mao, Wei Cai, Mark M Meerschaert, Mark Ainsworth, et al. What is the fractional Laplacian? arXiv preprint arXiv:1801.09767, 2018.

[78] SI Denisov, Werner Horsthemke, and Peter Hänggi. Steady-state Lévy flights in a confined domain. Physical Review E, 77(6):061112, 2008.

[79] Michael E Cates and Julien Tailleur. When are active Brownian particles and run-and-tumble particles equivalent? Consequences for motility-induced phase separation. EPL (Europhysics Letters), 101(2):20010, 2013.

[80] Alexandre P Solon, Michael E Cates, and Julien Tailleur. Active Brownian particles and run-and-tumble particles: A comparative study. The European Physical Journal Special Topics, 224(7):1231-1262, 2015.

[81] Michael Peskin. An Introduction to Quantum Field Theory. CRC press, 2018.

[82] Alexander Mikhailov and D Meinköhn. Self-motion in physico-chemical systems far from thermal equilibrium. In Stochastic Dynamics, pages 334-345. Springer, 1997.

[83] John D Weeks, David Chandler, and Hans C Andersen. Role of repulsive forces in determining the equilibrium structure of simple liquids. The Journal of chemical physics, 54(12):5237-5247, 1971.

[84] DM Heyes and JR Melrose. Brownian dynamics simulations of model hard-sphere suspensions. Journal of non-newtonian fluid mechanics, 46(1):1-28, 1993. 